\title{
Case Series: Handling of Fertilization Program in the Cervical Polyp Case
}

\author{
Ivanna Beru Brahmana ${ }^{1, *}$ \\ ${ }^{1}$ Obstetrics and Gynecology Departement Medical Study Program, Faculty of Medicine and Health Sciences, Universitas \\ Muhammadiyah Yogyakarta \\ *Corresponding author. Email: ivanna@umy.ac.id
}

\begin{abstract}
Pregnancy programs are carried out at the Obstetrics \& Gynecology clinic with infertility causes in the form of cervical polyp around $10 \%$. Provide a series of four cases of cervical polyps in pregnancy program patients. The first case occured in primary infertile 1.5 years, P0A0 27 yo, with irregular menstrual cycles. The examination showed cervical polyp. The second case occured in secondary infertile P1A1 33 yo, with complaints of vaginal discharge and odor. In these cases, the pregnancy occured during cervical polyps surgery and curettage of assistance. The third case was primary infertile 10 years, P0A0 33 yo. The examination showed vaginal discharge, cervical polyp, and erosion. The pregnancy succeds after four months by twisting cervical polyp, and assisting curettage. The fourth case was primary infertile 28 years, P0A0 54 yo. Gynecological examination showed vaginal discharge and cervical polyp. The infertile case did not have to be handled, but rather deal with vaginal discharge complaints. In the first and second cases with primary infertile 1.5 years and secondary infertile 12 years, successful pregnancy occured in the next month. The third case was the primary infertile 10 years and it was successfully conceived four months after the procedure. The fourth case concluded that the probable cause of infertility was due to undiagnosed cervical polyp. Handling of fertilization program in cervical polys case was successfull with twisting cervical polyps and accompanying curettage.
\end{abstract}

Keywords: cervical polyp, pregnancy program, twisting polyp

\section{INTRODUCTION}

The pregnancy program is an examination stage for infertile couples who want to have children as a form of their love. The term infertile is defined as a couple who has had a full year of uninterrupted sexual intercourse without contraception but has not yet given the result of pregnancy [1],[2],[3],[4]. Infertile is divided into two, namely primary infertile and secondary infertile. Primary infertile is when you have never had a pregnancy before. Meanwhile, secondary infertile is when you have had a previous pregnancy, even if the pregnancy did not end well, for example, abortion or ectopic pregnancy[2].

The prevalence of infertility increases from year to year. The current global prevalence of infertility ranges from 10 - $15 \%$ [1]. Masoumi S.Z., et al, 2015, distinguished the prevalence of infertility by $3.5-16.7 \%$ in developed countries, whereas in developing countries it ranged from 6.9-9.3\% 4. Infertility causes are divided into three categories: $22.8 \%$ due to male factors, $51.2 \%$ due to female factors, and $26 \%$ due to female and male factors[1]. Nardelli A.A., et al, 2014, stated that the causes of infertility in women were $35-40 \%$, in men by $20-40 \%$ and in men and women by $20-30 \%$, and the rest as unexplained infertility[3].

This paper reported a case series about the handling of the pregnancy program in cervical polyp cases. The patient managed to get her pregnancy after the procedure.

Infertility caused by the cervix's female factors is most frequently due to cervical polyp and cervical canal stenosis. The presence of cervical polyp can be identified by performing a gynecological examination and ultrasound. If a cervical polyp is found in the program the patient wishes to have, a polypectomy is immediately performed. After these actions, it is expected that the awaited pregnancy will succeed soon. If the stenosis is found in the cervical canal, dilatation with Hegar plugs may be needed. If pregnancy is not obtained naturally, for example, there is a cause of infertility also by male factors, then the intervention requires Intra-Uterine Insemination (IUI) to get the pregnancy[5].

Uterine or cervical factors account for at least $10 \%$ as a cause of infertility, such as a cervical polyp, cervical myoma, endometriosis, cervical stenosis, and uterine or cervical abnormalities[6]. According to Tanos V. et al, 2017, other literature mentioned that endocervical polyp occured in about $2-5 \%$ of cases[7].

\section{CASE SERIES}

\subsection{Case 1}

The married couple (wife was 27 years old and the husband was 28 years old). They married in 1.5 years, $\mathrm{P} 0 \mathrm{~A} 0$. The husband was as an entrepreneur, came to follow the pregnancy program. The patient had a history of irregular menstrual cycle lengths between 26-47 days. The patient had a history of previous examinations by getting an ultrasound examination without a gynecological 
examination. The husband had a Sperm Analysis examination with the results of Oligoteratospermia carried out.

At the first examination, it came an evaluation of the menstrual cycle, ultrasound examination, and gynecological examination. Ultrasound examination results showed normal uterus shape and size. The gynecological examination results showed that the cervix was intact, the ostium uterine external (OUE) was closed, no erosio porsiones were obtained, and a significant amount of vaginal discharge, and a $2 \times 2 \times 2 \mathrm{~cm}$ cervical mass was obtained. The patient was diagnosed as a 1.5 -year primary infertile with cervical polyp and leucorrhea. The patient was given therapy for leucorrhea in the form of single-dose Fluconazole for her and her husband. The wife was given additional therapy in the form of vaginal suppositories containing metronidazole and nystatin, once a day every night, for three consecutive nights. A diagnosis of cervical polyps was planned for cervical polyp twisting and accompaniment curettage.

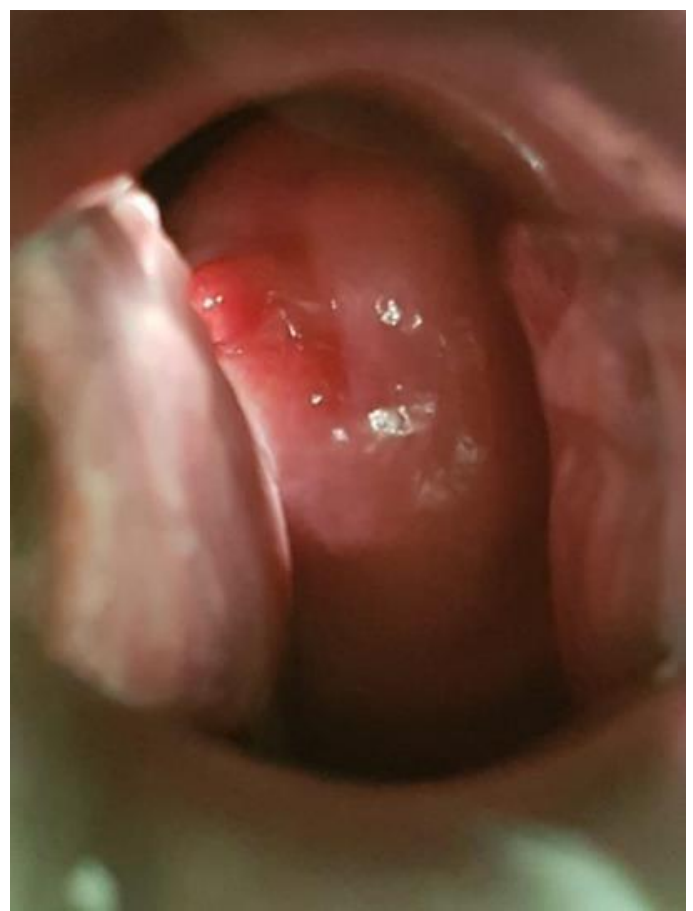

Figure. 1. Case 1: $2 \times 2 \times 2 \mathrm{~cm}$ in Size of Cervical Polyps

Cervical polyp twisting and accompaniment curettage were performed in the operating room using TIVA anesthesia. After the patient was anesthetized, field the operation in the vaginal area by showing the cervix. The mass of the polyp, which appears specularly, was lifted by twisting. Accompanied curettage was performed to clean the polyp mass and to minimize polyp regrowth.

The patient agreed to do cervical polyp twisting and accompaniment curettage. Patient preparation for polyp twisting and accompaniment curettage was performed. The general condition and vital signs of the patient were within normal limits. At the time of polyp twisting and accompaniment curettage, the patient's general condition was good. After the action, the general condition and vital signs of the patient were within normal limits. In posttreatment care on the ward, the patient's general condition and vital signs were within normal limits. The patient did not feel any complaints and smoothly urinated. The general condition of the patient allowed the patient to be discharged after treatment a day later.

The patient came to the clinic after three days of discharge from the hospital. The general condition and vital signs of the patient were within normal limits. The patient could defecate and urinate normally. The patient felts no complaints. In the gynecological examination, wound healing of cervical polyp wounds was obtained. The patient was allowed to go home and re-visit the clinic to discuss the Pathology Anatomy (PA) reading results. There was no prohibition on marital relations after the action due to the results of healing the former good actions.

One week later, the patient cre-visited with PA results. Pathological Anatomy results showed no malignancies and obtained endometrial polyp. The patient and her husband had given vitamin $\mathrm{E}$ therapy a $1 \times 1$ tablet for a month.

In the following month, the patient complained of not getting her period and delivered a positive pregnancy test result. In an ultrasound examination, the intrauterine gestational sac was obtained, according to the gestational age of 5 weeks. Furthermore, routine antenatal care (ANC) checks were performed on these patients. The pregnancy was in good condition and did not get complaints. Pregnancy condition followed the ANC schedule.

\subsection{Case 2}

A P1A1, 33 years old, came with complaints of leucorrhoea and smells. It sometimes bled from sexual intercourse. The last menstrual period (LMP) was on July 13, 2019. In term of obstetric history, the first pregnancy was abortion, and curettage. The second pregnancy was healthy by Caesarean Section (CS) 12 years ago, in which the baby was male with weight 3800 grams. The child was in good health. History of using birth control was denied.

Ultrasound examination results showed the normal size and shape of the uterus. The gynecological examination results showed that the cervix was intact, closed OUE, visible erosion portiones, vaginal discharge was found, and visible mass on the cervix measuring $3 \times 3 \times 3 \mathrm{~cm}$. The patient was diagnosed as a 12-year secondary infertile, P1A1 with cervical polyp and leucorrhea. Fluid-based cytology Pap Smear examination was performed on the patient. The therapy was given in the form of single-dose Fluconazole for each wife and husband. The wife was given additional therapy in the form of vaginal suppositories containing metronidazole and nystatin, once a day every night, for three consecutive nights. 


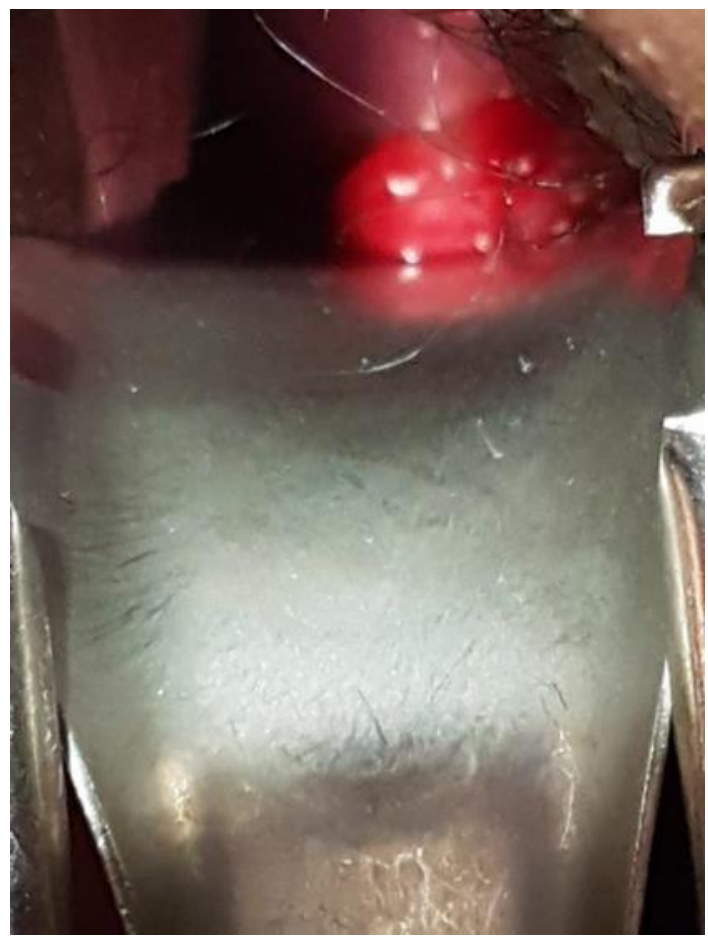

Figure. 2. Case 2: Cervical Polyps $3 \times 3 \times 3 \mathrm{~cm}$.

One week later, the patient revealed that the vaginal discharge reduced. Fluid-based cytology Pap Smear result showed that no malignancy was found, and non-specific cervicitis was found. The cervical polyp was recommended to conduct a cervical polyp twisting and accompaniment curettage in the operating room if the patient wanted another pregnancy.

After completing menstruation in August 2019, the patient agreed to do cervical polyp twisting and accompaniment curettage. The LMP was on August 13, 2019. The general condition of the patient before, during and after twisting the cervical polyp was good. The patient was discharged after twisting the polyp and curettage accompaniment, with a good general condition, no complaints of dizziness, nausea, and able to urinate smoothly. The patient was required to came to the clinic one week after discharge from the hospital or when the patient felt a complaint.

The patient came in October 2019, stating she was positively pregnant, with positive pregnancy laboratory results one month ago. The LMP was on August 13, 2019, currently the third pregnant patient with 11 weeks of gestational age. An ultrasound picture showed a single fetus, fetal heart rate (FHR) regular, according to 11 weeks' gestation. The patients was asked to perform ANC according to gestational age.

\subsection{Case 3}

A woman, a primary infertile of 10 years, P0A0 was 33 years old, came with a complaint intending the pregnancy program. The patient stayed at home together with her husband, so it was possible to have marital relations. There was no history of using contraception. The patient felt frequent vaginal discharge. Ultrasound examination results obtained form and size of the uterus within normal limits. The gynecological examination result showed that the cervix was intact, the OUE closed, erosio porsiones was found about one-third of the cervix, the vaginal discharge was found, and the cervical polyp was $3 \times 1 \times 1 \mathrm{~cm}$ in size.

The patient was given single-dose Fluconazole therapy for wife and husband respectively. The wife was given additional therapy in the form of vaginal suppositories that contain metronidazole and nystatin, once a day every night, for five consecutive nights.

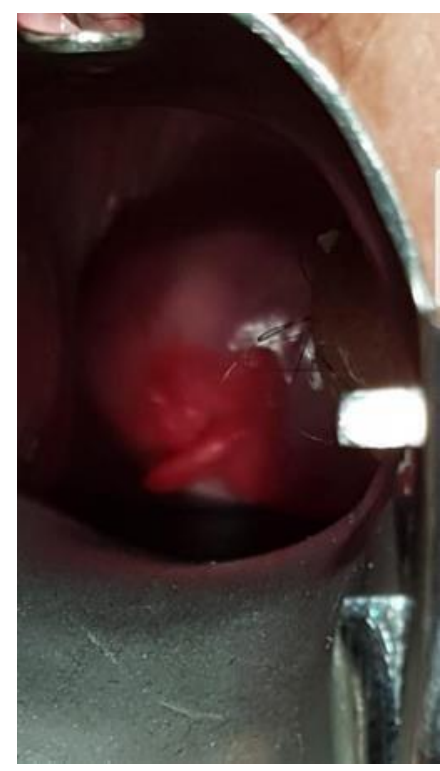

Figure. 3. Case 3: Cervical Polyps $3 \times 1 \times 1 \mathrm{~cm}$ with Erosio Portiones

Three weeks later the patient agreed to do cervical polyp twisting and cauterization to indicate the cervical polyp and erosio porsiones. The general condition of the patient was within normal limits. Before, during, and after the cervical polyp twisting and cauterization, the patient's vital signs were within normal limits. The patient was allowed to go home after feeling no complaints of dizziness, nausea, vomiting, and the patient could be spontaneous and normal urination. Five days after returning home, the patient came to the clinic, with no complaints. The results of the gynecological examination showed the cauterization wound showed a healing process.

In the following month, in February 2020, the patient came to the clinic, saying that she had menstruated twice after the procedure. There no complaints during menstruation. There was no difference compared to before the procedure. In the next two months the patient was still menstruating, and in the fourth month after the patient's actions came, she got a late mestrual cycle, with the LMP on April 20, 2020. The patient had conducted a pregnancy test with positive results in May 2020. At the time of the ultrasound examination, it showed a gestational sac (GS) $16.93 \mathrm{~mm}$ according to the gestational age of 6 weeks. The patient was asked to do routine ANC, and control for the 
next month.

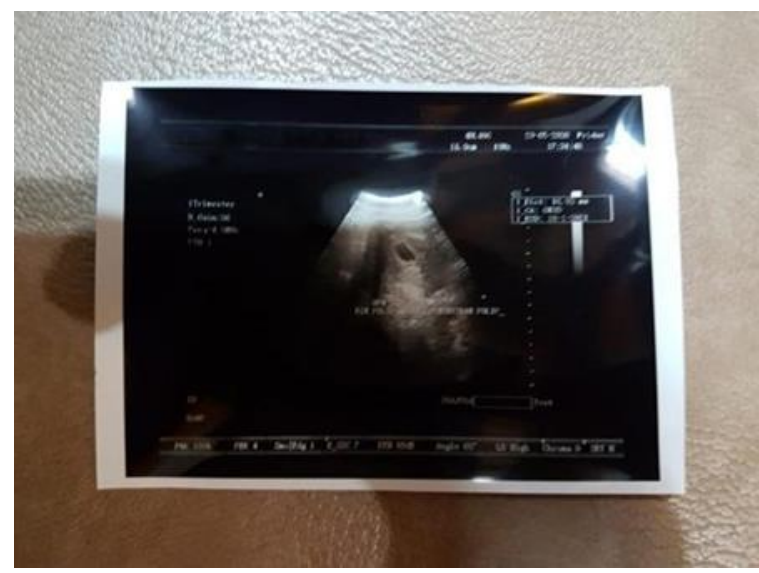

Figure. 4. Case 3: The Gestational Age of 6 Weeks

\subsection{Case 4}

A woman, primary infertile 28 years, P0A0 aged 54 years old, came with itching and smelling vaginal discharge complaints. The patient said that she had never used family planning, and had never checked herself about her infertile case. Ultrasound examination results showed the size and shape of uterine atrophy were within normal limits according to the age of menopause. Gynecological examination results showed vaginal atrophy with a narrowed vaginal canal, cervix atrophy, cervical polyp measuring $5 \times 3 \times 3 \mathrm{~cm}$, and vaginal discharge was found. In the patient's case, a vaginal swab was examined, and a week later, a positive Gram result was suspected of normal flora.

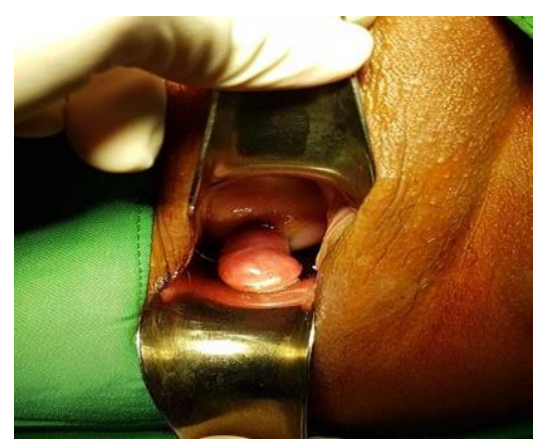

Figure. 5. Case 4: Cervical Polyps 5x3x3 cm.

The patient never checked for infertility. She accepted the condition that her pregnancy was because no pregnancy had been given. With the discovery of cervical polyps, as a result of the gynecological examination, it was suspected that this patient's infertility might be due to undiagnosed cervical polyp. The twisting polyp was performed on the patient, althought it was not to handle infertile cases, and the patient was given therapy for vaginal discharge complaints.

\section{DISCUSSION}

Cervical polyp as a cause of infertility in women, $90 \%$ grew exophytically, so readily identified with the gynecological examination. The remaining $10 \%$ developed intracervical, and the diagnosis was made by ultrasound examination. Management of cervical polyp with surgery in the form of polypectomy and cervical canal abrasion was performed under anesthesia. After polypectomy, patients usually obtained their pregnancy naturally, although some people needed an IUI to get their pregnancy. Intra-Uterine Insemination was needed for couples who also had infertility problems due to male factors. The cervical polyp provided recurrences, so observations should be made about 2-3 months after the polypectomy[5]. The diagnosis and action of cervical polyp twisting and accompaniment curettage in this case series are in line with the publication reported by Kunev A.K. Patients in the series of this case also underwent pregnancy naturally after cervical polyps were cleaned.

Cervical polyp disrupted sperm transport due to mechanical occlusion. Besides that, the accumulation of leukocytes around cervical polyp caused interference with sperm movement. Cervical polyp slowed down sperm transport so that bleeding easily arose when the polyp was touched, and in the presence of erythrocytes, it would stop the movement of sperm. Thus, cervical polyp caused infertility. After a cervical polyp was cleaned, pregnancy would naturally be obtained[5].

Pregnancy occured after cervical polyps were cleaned in the first, second, and third cases. The first and second cases showed their pregnancy in the following month, after polyp twisting. The third case showed pregnancy four months after the action. The first and third cases were P0A0 primary infertile 1.5 years and 10 years respectively, while the second case was a 12-year secondary infertile, P1A1 with a 12-year-old child born by CS.

The fourth case had never had a pregnancy, with P0A0, infertility 28 years. As her age was 54 years old, it was hardly possible to handle the infertile case.

This publication manuscript is based on research that has obtained ethical clearance number 1687/KEPUNISA/IX/2020, dated September 4, 2019.

\section{CONCLUSION}

Cases of cervical polyps as a cause of infertility were found at the time of the gynecological examination. With gynecological examination, the condition of the vagina and cervix could be displayed. The actions of cervical polyp twisting and accompaniment curettage were easy, simple, and could bring about a successful pregnancy, in the pregnancy program because of the cervical polyp. 


\section{LETTER OF ACCEPTANCE}

There is no conflict of interest in this matter. This manuscript was proofread and edited by UMY proofreader on November 24, 2020.

\section{ACKNOWLEDGMENTS}

This research was funded by the Faculty of Medicine and Health Sciences, Universitas Muhammadiyah Yogyakarta.

\section{REFERENCES}

[1] Moridi A., Roozbeh N., Yaghoobi H., Soltani S., Dashti S., Shahrahmani N., Mojdeh Banaei M., Etiology and Risk Factors Associated With Infertility, International Journal of Women's Health and Reproduction Sciences, 7(3), 2019, pp. 346-353.

[2] Anwar S and Anwar A., Infertility: A Review on Causes, Treatment and Management, Women's Health \& Gynecology, 2(6) 040, 2016, pp. 1-5.

[3] Nardelli A.A., Stafinski T., Motan T., Klein K., Menon D., Assisted reproductive technologies (ARTs): Evaluation of evidence to support public policy development, Reproductive Health, 11(76), 2014, pp. 1- 14.

[4] Masoumi S.Z., Parsa P., Darvish N., Mokhtari S., Yavangi M., Roshanaei G., An epidemiologic survey on the causes of infertility in patients referred to infertility center in Fatemieh Hospital in Hamadan, Iran J Reprod Med, 13(8), 2015, pp. 513-516.

[5] Kunev A.K., Diagnostics and Treatment of Cervical Causes of Infertility, J Biomed Clin Res, 12(1), 2019, pp. 33-39.

[6] Moramazi F., Roohipoor M., Najafian M., Association between internal cervical os stenosis and other female infertility risk factors, Middle East Fertility Society Journal, 23, 2018, pp. 297299.

[7] Tanos V., Berry K.E., Seikkula J., Raad E.A., Stavroulis A., Sleiman Z., Campo R., Gordts S., Review: The Management of Polyps in Female Reproductive Organs, International Journal of Surgery, 43(7e16), 2017, pp. 7-16. 